\title{
Stem Cell Technology in Regenerative Medicines and Cancer Treatment: Towards Revolution in Clinical Success
}

\author{
Muhammad Mukheed ${ }^{*}$, Alisha Khan', Husnain \\ Karim Riaz ${ }^{3}$, Irfan S ${ }^{3}$, Kainat Amjad², Hina Ilyas', \\ Aimen Afzal ${ }^{1}$, Shehreen Sohail ${ }^{3}$, Fareeha Sohail ${ }^{3}$, \\ Zoha $^{3}$, Raza MA ${ }^{2}$, Hayat $\mathbf{U}^{2}$, Khalid MU', Amna \\ Nahid $^{4}$, and Farooq $\mathbf{W}^{3}$ \\ ${ }^{1}$ Department of Biotechnology University of Gujrat, \\ Gujrat, Pakistan \\ ${ }^{2}$ Department of Biological Science University of Sialkot, \\ Sialkot, Pakistan \\ ${ }^{3}$ Department of Microbiology, University of Central \\ Punjab, Lahore, Pakistan \\ ${ }^{4}$ Department of Microbiology, Government College \\ University Lahore, Lahore, Pakistan \\ *Correspondling author: Muhammad Mukheed, \\ Department of Biotechnology, University of Gujrat, \\ Gujrat, Pakistan
}

Received: July 15, 2021; Accepted: August 03, 2021; Published: August 10, 2021

\begin{abstract}
Stem cells are undifferentiated, immature, and unspecialized cells having huge potential for differentiation and proliferation into the specialized functionalized cells. More recently, CSC has been described in breast cancer and brain tumors where they make up as few as $1 \%$ of the cells in a tumor. The features of cancer stem cells are just like normal stem cells but their replication rate many times faster than normal cells. Regenerative medicines are based on stem cells, are potentially useful to regenerate damaged cells, tissues, organs and replace cancer cells with normal cells. Induced pluripotent stem cells are the most important candidates for regenerative medicines, tissue engineering, cell reprogramming, and 3D printing. Cancer Stem Cells (CSCs) have a tumorinitiating capacity and play crucial roles in tumor metastasis, relapse and chemo/ radioresistance. Because CSCs are resistant to chemotherapeutic drugs and cause recurrence of cancer and also have the ability to be regenerated; they can cause serious problems in the treatment of various cancers. Numerous biocompatible biomaterials, miRNAs, nanomaterial, artificial intelligence, and machine learning are uses to reprograms stem cells into regenerative medicines for the treatment of cancer. The present paper describes the applications and importance of stem cells in regenerative medicines, cancer stem cells targeting therapies, and the role of miRNAs in cancer stem cells targeting.
\end{abstract}

Keywords: Cancer stem cells; Induced pluripotent stem cells; Regenerative medicines; Tissue engineering; Biomaterial; miRNAs; Artificial intelligence

\section{Introduction}

The stem cells are immature and unspecialized cells that have robust self-renewable potential and the ability to differentiate into all mature and specialized cells. These cells can replace the damaged and defective cells and play important role in the regeneration of damaged body parts. The role of stem cells in regenerative medicines and therapies has been widely anticipated [1,2]. The adult bone marrow stem cells can replicate repeatedly and differentiate into specialized mesenchymal tissues such as bones, cartilages, muscles, tendons, and others. The self-renewable ability and potential to differentiate into all cells evaluate the importance of stem cells in regenerative medicines and tissue engineering. Stem cells are isolated from the patient body and expand by ex vivo culturing and by combining with biodegradable and biocompatible inserted into the patient body where they grow normally to repair damaged or injured cells and tissues [3]. Human adipose tissue-derived stem cells have ideal applications in tissue engineering and cancer treatment. Adipose-derived stem cells are more beneficial and reliable than mesenchymal stem cells because these cells are easily isolated and have huge potential to differentiate into osteocytes, hepatocytes, adipocytes, cardiomyocytes, pancreatic beta cells, neural and endothelial cells. Low immunogenicity, secretion of trophic factors, and immunosuppressive properties of ADSCs make them more relevant and preferable in regenerative medicines and cancer treatment [4]. The human-induced pluripotent stem cells assemble with embryonic stem cells in reprogramming and self-renewable characteristics and use cardiac research from the last ten years. hiPSC derived cardiac myocytes are a potential platform for the human disease model and an important tool for drug delivery and testing. In advance, hiPSCs are a common source of stem cells in human-animal regenerative models because these cells capitulate phenotypic differences due to genetic variations [5]. The glioblastoma contains a rich amount of tumor-causing cancer stem cells. These cells make a contribution to tumor propagation and therapeutic resistance but the mechanism is not known until. According to recently performed studies the CD36 expression was noted in GBM which are rich in cancer stem cells and when these CD36 are expressed with CD133 and integrin alpha 6 their selfrenewable and tumor initiation capacity declined. Moreover, it has been confirmed that the ligands of CD36 are oxidized phospholipids present $n$ GBM and the proliferation of CD36 decreases when exposed to oxidized low-density lipoproteins. Hence the enhanced expression of scavenger receptors provides survival and metabolic advantages [6]. Scientists have strong shreds of evidence that cancer cases are increases due to the transformation of normal cells into cancer cells. Sometimes after conventional therapies cancer cells again proliferate and tumors formed. This chemoresistance affects cancer stem cells arise due to the activation of $\mathrm{B}$ cell-specific Moloney murine leukemia virus integration site 1 (BMI1). BMI1 has an important role in the cell cycle, regulation, and proliferation of normal and cancer stem cells. It is also suggested that BMI1 are present on cancer stem cells and after therapy, these factors are reactivated and form tumor again 
[7]. Several pluripotent transcriptional factors such as SOX2, Nanog, KLF4, OCT4, and MYC are potential biological activators of cancer stem cells. It is also confirmed that several intracellular signaling pathways including Notch, JAK-STAT, Wnt, TGF, PPAR, and $\mathrm{P} 13 \mathrm{~K} / \mathrm{AKT} / \mathrm{mTOR}$ and extracellular factors hypoxia, vascular niche, cancer-associated fibroblast, cancer-associated MSCs, and exosomes are proliferator and regulator of cancer stem cells [8]. Due to these abnormalities, regenerative medicines are preferred to treat cancer because regenerative medicines are based on stem cells and replace the tumor-causing cells with normal cells.

\section{Personalized Regenerative Medicines}

Personalized medicine is referred to as patient-specific therapeutic agents. Regenerative medicines and cell therapy are depending on cells-based products to personalized medicine. Mesenchymal stem cells, embryonic stem cells, and induced pluripotent stem cells are useful in targeted therapies. Due to fewer immune rejection chances, induced pluripotent stem cells have been introduced as a therapeutic candidate for personalized regenerative medicines. It also noted that multiple factors such as recipient factors, donor factors, and overall body environment for stem cell activation are important to achieve personalized stem cell therapy. A lot of technologies such as molecular diagnostic tools play important role in the development of regenerative medicines. Embryonic stem cells have enormous potential in regenerative medicine but because of highly immune rejection chances and ethical issues these are prohibited and somatic cells are reprogrammed in culture media and pluripotency induced [9]. In culture media, somatic cells are undergo reprogramming by reprogramming factors such as Nanog, Oct4, Sox2, and Lin28 [10]. Due to self-renewable and potential to differentiate into all cell kinds, stem cell provides a significant platform for regenerative medicines. Human induces pluripotent stem cells are used in the treatment of cancer, cardiovascular, renal, kidney, neurological disorders, Parkinson's disease, and spinal cord injury act. It has been illustrated that human adipose-derived stem cells are a kind of progenitor stem cells and a major source of pluripotent stem cells. ADSCs are also substituted for bone marrow MSCs as a secondary source of regenerative medicine [11]. The mesenchymal stem cells are adult tissues that undergo multilineage differentiation when cultivated and expand in-vitro. Groups of researchers have been derived MSC from iPSCs (iPSCs-MSC) as an alternative source of MSCs personalized regenerative medicine and also applied in direct cell therapy, gene therapy ad tissue engineering [12]. The experimental studies are conducted to generate cardiomyocytes from iPSCs by inducing embryonic body formation and treatment with bone morphogenic protein 4, Activin A, and inhibition of Wnt signaling. These derived $\mathrm{CMs}$ are heterogeneous, need maturation and purification for in vitro CMs mimicry. Mature CMs are used in regenerative medicines by developing tissue-engineered cardiac patches [13]. The human body loses tissues and organs in trauma, disease, and defects. As the human body has a very low potential to regenerate damaged organs and tissues as compared to amphibians and due to the shortage of organs for transplantation scientists need to develop reliable source organs and tissues. Tissue engineering and regenerative medicines are employing biological and engineering to create new organs and tissues via three-dimensional bioprinting. Stem cells and scaffold of specific organ in a specific culture media will develop into required organ or tissue. 3D bioprinting is the most advanced tool in personalized regenerative medicines and tissue engineering [14]. Biological wastes such as urine are also inhabitants of cells that have the self-renewable ability and differentiation potential which originally derived from nephrons, renal pelvis, bladder, and ureters. The urine-derived stem cells are phenotypically mimicking with bone marrow mesenchymal stem cells and reprogrammed into an induced pluripotent stem cell. These urine-derived iPSCs have potential use in personalized regenerative medicines, tissue engineering, and drug delivery. For example, bone marrow MSCs are not differentiated efficiently into the urothelial cell so UDSC-iPSCs have better performance and huge potential for urothelial cell production. Urine-derived stem cells are also used in bone regeneration, neural cell regeneration, and the treatment of prostate cancer [15]. The genome editing tools such as zinc finger nucleotide, TALEN, and CRISPR Cas9 system has important application in tissue engineering and personalized medicines. When tissues, organs, or cell lines are proliferating in vitro, cell proliferation and reprogramming are enhanced by altering the expression rate of specific genes. According to recently performed studies, it is confirmed that CRISPR, CRISPRi, and CRISPRa are mostly used to edit gene or regulating gene expression for bone regeneration, neural cell regeneration, and treatment of prostate cancer, leukemia, skin cancer, and other diseases [16]. As we describe above iPSCs are specific kinds of stem cells and have huge potential in personalized medicine because these cells are ethically accepted and following reprogramming injected back into damaged tissue or organ by vectors such as retrovirus and lentivirus. Sometimes reactivation of viral transgene due to incomplete silencing and interfering with induced iPSCs proliferating potential cause tumorigenesis. So, retrovirus and lentivirus use in the clinical field is prohibited. Hence non-viral tools for injection of Yamanaka factors (Sox2, Oct4, c-Myc, and Klf4) are developed such as RNA or DNAbased electroporation and polymer or lipid-based nanoparticles. As small-sized, high surface area and lipid-based nanoparticles are not interacting with the host cell genome, they are employed to deliver reprogramming factors on specific sites [17]. Recently a technology named direct cell reprogramming or transdifferentiation was developed in which one somatic cell is directly programmed into another cell without induction of pluripotent state. Direct cell reprogramming can differentiate abundant cells in the body into desired tissues or cells therefore this technology has an advanced role in regenerative medicines. Several cells such as neuronal cells, skeletal myocytes, and more which have the potency to regenerate damaged tissue or cells, replace cancerous cells are generated through direct cell reprogramming [18].

\section{Cancer Stem Cell-Targeted Therapies}

Cancer stem cells are a subpopulation of cells that have similar differentiation, self-renewable, and proliferation activities as normal somatic and embryonic stem cells. Multiple surfaces and enzymatic markers are characterized for the identification of cancer stem cells such as CD34+, CD38- and cancer stem cells express multi-drug resistance proteins and upregulated gene expression. There multiple classes of cancer stem cells such as breast cancer stem cells, brain cancer stem cells, leukemia stem cells [9]. There are several cancer celltargeted therapies. One of the most important signaling pathways is the Notch pathway which is revealed as the target for different kinds of 
tumors. The activation of notch occurs when ligand bind with notch receptor which followed cleavage via ADAM protease and gammasecretase and results in exposure of notch intracellular domain. This NICD transport to the nucleus where it binds with cancer stem cells transcription factors and becomes converted into an activator of the Notch gene. The use of gamma-secretase inhibitors and antibodies is successful to treat breast, pancreatic, and renal cell carcinoma [19]. It has been noted that the complicated phenotypes and biological behavior make it difficult to purify cancer stem cells and further culturing because cells may lose their stemness. Nowadays multiple receptor/markers, cancer stem cells related factors are recognized to purify and therapeutically target CSCs. Some important cancer stem cells related markers are ABCG2, ALDH1, CD133, CD9f, OCT4, OPN, and SOX2. These stem cell markers provide an accurate and easy approach to identify cancer stem cells [20]. Recently performed studies proposed a dual-targeting strategy to target cancer stem cells. In this strategy, the agent VS 5584 is used as a dual inhibitor of $\mathrm{mTORC}^{1} 1 / 2$ and class IPI 3-kinase which results in inhibition of tumor development and CSCs loss their ability to form tumors [21]. The cancer stem cells are developed resistance against conventional therapies because CSCs are localized in a microenvironment named "vascular niche". This niche has a major role in resistance against conventional therapies. This problem force scientists to move towards intrinsic and extrinsic factors-based targeting. These therapies are based on DNA repairing systems, Scavengers, and multiple drug transporters $[22,23]$. In a recently performed study it is proved that the milk protein alpha casein when overexpressed in Triple-Negative breast cancer lines, the tumor developing activity of cancer stem lines declines because the alpha casein protein inhibits the expression of STAT3 and HIF-1alpha. Moreover, the alpha casein protein also reduces the expression of cancer-associated fibroblasts [24]. Cyclopamine a sterile like compound is bind and inhibits SMO protein by competing for binding with Vitamin $\mathrm{D}$ which in turn depress the tumor formation ability of cancer stem cells. It is also investigated that cyclopamine compounds reduce the cancer cell proliferation in prostate and gastric cancer cell lines [25]. Targeting cancer cell surface proteins with antibodies, $\mathrm{ABC}$ transporter inhibition, and ALDH enzymes along with small molecules are useful in tumor reduction. As we know that lung cancer cells multiple markers, one is CD133. CD133 marker in association with OCT4 gene. The high expression of OCT4 induces self-renewable ability in CD133 marker cells. The siRNA inserts to knock down OCT4gene expression which results in loss of the self-renewable ability of CD133 cells and tumor formation activity suppressed. Alternatively, it is suggested that $\alpha 6 \beta 1$ integrin is involved in the genesis of cancer stem cells and helps in cancer cells' survival of CSCs. By targeting integrins the cancer stem proliferation can be prohibited [26]. The GSK3 $\beta$ is involved in cancer pathways especially it is upregulated in breast cancer. The high expression of GCK3 $\beta$ has a pivotal role in the survival of Triple Negative Cancer. Inhibition of this pathway decreases the cancer cell markers proliferation and stemness of cancer stem cells. The Wnt$\beta$-catenin pathway inhibits via GSK3 $\beta$ which play important role in tumor suppression [27]. Overexpression of proliferative pathways in cancer cells is in association with tyrosine kinase activity of membrane receptors and cytoplasmic proteins. Tumor cells with overexpression of EGFR receptors are targeted by the application of EGFR tyrosine kinase inhibitors. In the same way in breast cancer, HER2 receptors are overexpressed and the employment of HER2 inhibitors reduces proliferation and differentiation of these markers. HER2 receptors have an important role in the maintenance of stemness in cancer stem cells [28].

As we described earlier, the proliferation and self-renewable activity of cancer stem cells are responsible for resistance against chemo and radiotherapies. The cancer stem cells also have a huge capacity for DNA repairing which also contributes to resistance. In glioma CD133+ greater expression of DNA repair checkpoints is present. The damaging or inhibition of these checkpoints leads to a reduction in tumor formation. Target eliminations of cancer stem cells take part in the inhibition of tumor generation. CSCs have many surface markers, providing a platform to develop strategies that directly target these markers [29]. Recently a group of scientists proved that Chimeric antigen $\mathrm{T}$ cells receptors have an immunotherapeutic approach to target cancer cells. The chimeric $\mathrm{T}$ cells are command to express artificial receptors which have a targeting domain from antibodies connected with an intracellular domain. The antibodies responsible for targeting surface antigens on CSCs are the origin of chimeric antigen receptors [30]. Natural killer cells have revolutionized the ability in lyses of cancer stem cells. By increasing the expression of natural killer cells ligands as NKp30 and NKP44 scientists improve the cytotoxic activity of NK against CSCs [31]. According to recently conducted studies, melatonin decreases colony formation, invasion, proliferation, and migration of melanoma cells and promotes cell arresting, stemness decreasing in cancer stem cells by stimulating vemurafenib. By repudiating the nucleus translocation of NF-kBP50/p65 and bind it with promoters named iNOS and hTERT, melatonin intensifies the anti-tumor effect of vemurafenib [32]. Epigenetic alterations have a significant and synergistic role in gene expression and useful in carcinogenicity. Important and specific pathways as DNA methylation, regulatory RNAs, and chromatin remodeling are organized in cancer cells especially via epigenetic mechanisms. Through epigenetic mechanisms, phenolic compounds for example polyphenols having anticarcinogenic, anti-tumor, antiinflammatory, and anti-oxidant activities, neutralize and suppress cancer stem cells proliferation, development, microenvironment, and their metabolisms. Hence use of these phenolic compounds is helpful in the treatment of different types of cancers [33]. According to research published in 2019, the NRG fusion proteins are drivers for several cancers as pancreatic, lungs, prostate, and breast cancers. The NRG1 fusion protein binds with HER3 and causes heterodimerization of HER $2 / 3$ which leads to increasing downstream signaling pathways and tumor growth. HER2 and HER3 directed antibody called MCLA128 is applied directly on cancer stem cell lines. MCLA-128 blocks the binding of ligand to HER3 and decreases downstream signaling and tumor growth shrink [34]. Moreover, a synthetic guanosine analog and antiviral molecule Ribavirin used against hepatitis $\mathrm{C}$ also have an anti-tumor capacity. The transcription factors eLF4E are inhibited by ribavirin in leukemia and lymphoma. These factors have an important and crucial role in RNA export and expression of oncogenes such as MYC, BCL2, and BCL6. Ribavirin directly targets two complexes eLF4E and IMPDH. In addition, ribavirin also indirectly targets carcinogenic pathways including EZH2 and MAPK pathway and reduces the tumor burden on cancer cell lines [35]. A nonsteroid and antiandrogen agent enzalutamide is demonstrated as a targeting agent in prostate cancer and androgen receptors activity in castrate 
Table 1: Cancer stem cells targeting therapies.

\begin{tabular}{|c|c|}
\hline Mechanism & Targeting Agents \\
\hline Notch Signaling Pathways & MK-0752 [38,39], OMP-21M18 [40] \\
\hline Wht Signaling Pathways & PRI-724 [41] \\
\hline P13K Pathways & BGT-226 [42] \\
\hline CD44 & H90(anti-CD44 mAb) [43] \\
\hline MAPK, EZH2 Pathways & Ribavirin [44] \\
\hline HRAS Mutations & Type of Cancer \\
\hline Targeting Agents & Acute Myeloid Leukemia [45] \\
\hline MK-0752 & Breast Cancer [46] \\
\hline MK-0752-docetaxel & Esophageal Cancer [47] \\
\hline PKM-01 & Pancreatic Ductal Carcinoma [48] \\
\hline Vantictumab & Chronic Lymphocytic Leukemia [49] \\
\hline Cirmtuzumab & Chronic Lymphocytic Leukemia [50] \\
\hline Ibrutinib & \\
\hline
\end{tabular}

resistance prostate cancer. As we know that JAK2 and STAT5 mRNA levels are in peaks in prostate cancer. This evaluated level of oncogene mRNAs is inhibiting by enzalutamide [36]. Recently a new antitumor agent tipifarnib is recognized as the target agent of tumors with HRAS mutation. HRAS is confessing as an oncogene and overexpressed in squamous cell carcinoma. The activity of HRAS is synchronized by the enzyme farnesyltransferase. Tipifarnib inhibits this enzyme and results in decreasing HRAS expression and tumor growth diminished but until tipifarnib in trial phases [37-39]. Many therapies are in trial phases, some are in use but these therapies are not completely treating cancer. Now the revolutionized steps are taking to target cancer stem cells. From last decades it hypothesized that regenerative medicines are accomplished in cancer stem cells targeting. Induced pluripotent stem cells with genome editing have a mountain approach for cancer stem cells targeting. The cancer stem cells targeting therapies, their mechanism and targeting agents are listed in Table 1.

\section{miRNA Therapies in Regenerative Medicines and Cancer}

miRNAs are non-coding RNAs containing 20-24 nucleotide sequences and have a role in post-transcriptional modifications. miRNAs are clinically important because of their impact on metabolism, differentiation, proliferation, and apoptosis. Several studies have confirmed the supremacy of miRNA on several diseases such as cancer, neurological disorders, and cardiovascular diseases [51]. More than $60 \%$ of human genome expressions are regulated by miRNAs especially associated with human cancers and regulation of stemness characters in cancer stem cells. The miRNAs effects and target the oncogene signaling pathways that contributed to the maintenance of stem cell features like self-renewable, regeneration, proliferation, and differentiation of cancer stem cells. miRNAs have a major influence on cancer stem cells signaling pathways such as notch, BMI-1, and Hedgehog pathways [52]. In breast cancer, the cancer cells have radioresistance ability and some miRNAs are also involved in cellular response against radiotherapy. These miRNAs act as a radio response biomarker in breast cancer stem cells. The miRNA are biomarkers for cancer cell detection but available methods are insufficient for miRNA detection in cancer stem cells. For the detection, scientists combined duplex-specific nuclease strategy, liquid chromatography, and tandem mass spectrometry. A probe with substrate peptide with reporter peptide and tryptic cleavage site with biotinylated DNA sequence which is a complement to target miRNA is designed. This DNA-probe peptide hybridizes with target miRNA and duplex-specific nuclease break probe and target miRNA. Released miRNA signal amplifies by mass spectrometry at a range between $1 \mathrm{fM}$ and $200 \mathrm{Fm}$. This miRNA 200c is quantified as a biomarker in breast cancer [53]. There are several types of miRNAs for example miR-22 and miR-99 are oncogenic miRNAs and regulate cancer stem cell replication. The miR-126 control cell cycle in leukemia cancer cells, miR-34a inhibit c-Met and notch $1 / 2$ pathways in glioblastoma cancer stem cells by binding with 3'-UTR regions. The miR-200, miR-221, miR-137, and miR-203 target many genes and responsible for the proliferation, regulation, and differentiation of CSCs [54]. Moreover miR-711 cause downregulation of CD44 expression and inhibit epithelial to mesenchymal transition by downregulating vimentin protein expression and upregulating E-cadherin protein expression in gastric cancer [55]. The miRNAs are therapeutic agents in cancer treatment. Their features are inhibiting by the transfection of miRNA inhibitors such as antimiRs, antagomiRs, and miRNA sponges. A simple mechanism of inhibition is that a single strand anti miRNA oligonucleotide complements to target miRNA, bind it and block its binding with 3'-UTR regions. AntagomiRs are cholesterol conjugated and 2O'mrhtylated single-stranded RNAs and sponges restrain miRNAs presentation by binding competitively to miRNAs [56]. It has been suggested that by delivering miR-210, the exosomes derived from gemcitabine resistance pancreatic cancer stem cells mediate the exportation of drug resistance traits into GEM-sensitive pancreatic cancer stem cells. The miR-210 has an important role in GEM resistance which is the first-line treatment of pancreatic cancer. The miR-210 has a tremendous role in declining cell cycle arrest at the G2/M phase, inhibits GEM-induced apoptosis, and stimulates Bxs tumor growth rate [57]. The previous experiments have been results in findings regulation of miRNA in CD133+ cancer stem cell where they regulate CD133 expression as tumor suppressor or promoter. The miR-181a upregulates as a tumor suppressor, inhibits invasion, proliferation, and induced apoptosis in glioma cancer cells by targeting Notch pathways. The miR-107 and miR-139 are tumor suppressor genes, inhibits CD44+ and CD133+ cell expression, inhibits notch signaling pathway, and suppress drug resistance against tumors. Furthermore, miR-34c upregulated in prostate cancer and block the self-renewable capacity of CD133+ cells and miR-200c is also a tumor inhibitory factor in glioma cancer cells, inhibit tumor growth by targeting notch and CAMP pathways [58]. Because of resistance against drugs, chemo, and radiotherapies the cancer stem cells are not destroyed. Scientists and researchers move toward targeting CSCs at a molecular level and transcriptional level. These cancer stem cell targeting strategies provide vast opportunities to control cancer at initial stages. Along with micro-RNA-based therapies, several biomaterials are in clinical use to treat cancer stem cells. The miRNA therapies open a new advanced era in the treatment of various cancers. It is recognized that miRNA biomarkers use for diagnosis, classification, and treatment of oncogenes. Micro RNA mimics and miRNA antagonists are inhibiting and suppress oncogenes and restore the expression of tumor suppressor miRNAs [59]. Some important miRNAs in cancer therapies are listed in Table 
Table 2: Tumor's suppressor miRNA and their target genes.

\begin{tabular}{|c|c|c|}
\hline miRNA & Type of Cancer & Target Genes \\
\hline $\operatorname{miR}-9,-125 a,-125 b[60]$ & Neuroblastoma & $\begin{array}{c}\text { Tropomyosin-Related } \\
\text { Kinase }\end{array}$ \\
\hline miR-10b [61] & Breast Cancer & HOCD10 \\
\hline miR-17-5P, -20 [62] & Breast Cancer & AlB1 \\
\hline miR-16-1, -15a [63] & $\begin{array}{c}\text { Chronic lymphocytic } \\
\text { leukemia }\end{array}$ & $\mathrm{Bcl} 2$ \\
\hline miR-19 [64] & Colon Cancer & Thrombospondin-1 \\
\hline miR-21 [65] & Hepatocellular Cancer & PTEN \\
\hline miR-34a [66] & Neuroblastoma & $\mathrm{E}_{2} \mathrm{~F}_{3}$ \\
\hline miR-127 [67] & Bladder Cancer & $\mathrm{Bcl} 6$ \\
\hline miR-206 [68] & Breast Cancer & ER alpha \\
\hline miR-221, -222 [69] & Prostate Cancer & P27 \\
\hline
\end{tabular}

2.

\section{Biomaterials in Regenerative Medicines and Cancer Therapies}

The regenerative medicines have huge potential to repair and regenerate damaged or diseased cells, tissues, and organs of the human body using designed biocompatible, biophysical, and biochemical characters biomaterial. The inorganic biomaterials regulate cell to cell and cell to matrix interactions. The biological characters of biomaterials are improved and upgraded by the addition of inorganic micro and nanoparticles to polymeric networks. Inorganic biomaterials released bioactive ions induce microenvironment in cells to heal and regenerate damaged cells, tissues, and organs [70]. Zinc-based biomaterials also have biodegradable, biocompatible, and regenerative features. Importantly used zinc biomaterials are zinc ceramic nanoparticles, metallic zinc alloy, and zinc metalorganic frameworks. Due to the larger surface area zinc metallic organic frameworks have ideal applications in drug delivery and cancer therapy. Nanostructure and antibacterial properties made zinc ceramic nanoparticles useful in tissue engineering and cancer therapy [71]. Recently natural polysaccharide chitosan derived from chitin is identify as a good candidate in tissue engineering. It bears a free amino acid group $\mathrm{n}$ backbone chain that undergoes further chemical modification for developing different biomaterials in tissue engineering. In addition, starch, cellulose, alginate, collagen, and hyaluronic acid are naturally abundant polymers with major applications in regeneration and tissue engineering. Chitosan is used for healing wounds, regeneration of bone and cartilages. In drug, delivery chitosan is used as a nano or microparticle and vehicle for drug delivery in nasal, buccal, ocular, and pulmonary tissues. Moreover, it also uses as a non-viral gene delivery system [72]. Several studies have been confirmed that biomaterial-based scaffolds such as polymeric-based scaffolds, solid lipids, and metallicbased scaffolds are successful in the regeneration of lung tissues. Nanotechnology provides extraordinary opportunities to enhance tissue engineering and regeneration medicines developments [73]. Polyphosphazene is generally accepted as a new candidate for tissue engineering in combination with other polymeric compounds. Neutral $\mathrm{pH}$ of polyphosphazene made the ideal and unique class of biomaterials as compare to other ones. Structurally this polymer has an inorganic backbone with alternating nitrogen and phosphorus and side groups are commonly amino acids and peptide esters. The side groups are further modified to generate biomimetic scaffolds that act as a template for the regeneration of tissues or organs. Numerous clinical laboratories use linear polyphosphazene and cyclo-matrix polyphosphazene in drug, gene, and protein delivery [74]. Both natural and synthetic biomaterials are used in intestine regeneration. Collagen and chitosan are natural polymers used for the small intestine, colon, and circular smooth muscle regeneration. In the same way, synthetic polymers such as PGA, PLLA, PLGA, and PCL are good employees for small intestine regeneration in patients with intestinal failure. Moreover, these synthetic polymers are useful in gene delivery and targeted drug delivery in cancer treatment [75]. Because of difficulties in targeting cancer cells, scientists move towards cell therapy in which mesenchymal stem cells, embryonic stem cells, induced pluripotent stem cells, T cells delivery, and islets transplantation have a prodigious role. The performance of cell therapy is enhanced by patient-specific, biocompatible, and biodegradable biomaterials as they provide $3 \mathrm{D}$ organization and scaffolds for organ or tissue engineering. Alginate, cellulose, and HEM-MMA are polymeric biomaterials used for microencapsulated cell-based therapies [76]. Recently nitric oxide is recognized as an important signaling molecule and plays a pivotal role in the modulation of stem cell behavior such as proliferation, differentiation, and survival. Biomaterials-based therapies that are utilizing nitric oxide generation support stem cell delivery and it is a collaborative approach in improving tissue engineering. Cancer stem cells' microenvironment is regulating by using nitric oxidereleasing biomaterials [77]. In cancer immunotherapy, the patient's immune system is regulated and stimulate to target cancer stem cells. Numerous immunotherapeutic approaches are designed but have little performance. Biomaterials-based immunotherapies can successfully educate the immune system to target cancer stem cells. In $3 \mathrm{D}$ bioprinting, these biomaterials use as scaffolds to regenerate the heart, lungs, kidneys, eyes, and other degenerate organs of the human body. As biomaterials are biocompatible, their characteristics meet patient body characters and having lower immune rejection chances, biodegradable and consensus physiochemical properties with the human body. These characteristics made them revolutionized, ideal and unique candidates for regenerative medicines, tissue engineering, and cancer stem cells targeting. Biomaterials in combination with radiotherapy trigger immune therapy [78].

\section{Nanotech Assisted Regenerative Medicines}

Nanotechnology deals with materials at the nanoscale and it brought modern revolutions in regenerative medicine and tissue engineering. Nanomaterials along with an advanced level of genomics and proteomics opened a bright future of regenerative medicines and tissue engineering. Due to the small size and larger surface area nanomaterials have huge potential for drug delivery, gene delivery, and synthesis of scaffolds for organ regeneration [57]. In the current era, 3D printing and nanotechnology are important and modern tools in personalized regenerative medicines. Semisolid extrusions, tissue engineering, cell growth, and inorganic nanoparticles are the most dedicated 3D printing technique, substituents/agents [79]. Nanoparticles-based drugs provide a lot of opportunities for diagnosis, treatment, and cure of cancer, epilepsy, kidney stones, and other challenging diseases. A variety of nanostructures are designed with stealth properties in diagnosis, imaging, and drug delivery by 
manipulation of small size, larger surface area, surface characteristics, and surface modifications. The nanoparticle-based drug can easily cross the blood-brain barrier, directly target diseased cells and tissues and easily handle. So, these confidential characters made nanoparticles more revolutionized in regenerative medicines [80]. In recent past years, it is conducted that electrospinning fabricates are nano dimensional fibers having porosity, high surface area, and capacity to incorporate biocompatible materials into nanofibrous polymeric structures. The bio adaptable nanoscale electro-spun fibers have broadspectrum applications in tissue engineering, regenerative medicines, implants, drug delivery, and biosensing [81]. Nano carrier-based delivery platforms have confidential applications in cancer treatment. Gold nanoparticles have enhanced permeability and retention effect properties. These are accumulated in cancer cells and restrict cancer metastasis. Gold nanoparticles are used to target cancer cells, diagnose cancer, imaging cancer cells/sites, as functionalized agents for cancer treatment, and treat prostate cancer [82]. Nanoparticles used in tissue engineering are classified into two categories biodegradable and nondegradable nanoparticles. Biodegradable nanoparticles including chondroitin sulfate, collagen, PLA, PLGA, polycaprolactone, and HA whereas mesoporous silicon dioxide, gold nanoparticles, quantum dots, lipids, and SPIOs fall into the nondegradable nanoparticles class. These nanotechnological materials are used in osteochondral regeneration [83]. Cell reprogramming along with grammatical advances in biomaterial and macro or nanoparticles is a decisive tool in the engineering of stem cells fate, regenerative medicines, disease modeling, drug and gene delivery. A great number of inorganic and hybrid nanoparticles i.e., mesoporous silica, calcium phosphate, graphene oxide, and gold-based nanoparticles are invented for the stimulation of cell reprogramming. As a consequence of its small size, high surface area, biocompatible capability, and functionalization capability, mesoporous silica is broadly used as a nanocarrier for drug and genome delivery [84]. Rather than regenerative medicines and tissue engineering, bioimplant engineering is established to recover damaged tissues and organs. Nanomaterials have huge potential to influence surface properties regarding surface energy, surface chemistry, and surface topography. These properties made nanomaterials a good candidate for cell reprogramming, tissue engineering, and bioimplant engineering. It has been tremendously investigated that nanoparticles-based biomaterial polish up cell adhesion, differentiation, proliferation, and migration of bioimplants in the orthopaedical platform. Orthopaedical biomaterials are classified into classical biomaterials which include metallic, nonmetallic, and their alloys, and nanophase biomaterial which are nanophase metals and nanophase nonmetals [85]. A lot of organic nanoparticles as lipid solid nanoparticles, liposomes, and micelles are broadly used in drug delivery, gene delivery, and cancer diagnosis. Lipids-based nano drugs ThermoDox and Doxil are using in the treatment of hepatocellular carcinoma and Kaposi's sarcoma. Both drugs are temperature sensitive and approved by FDA [86]. Cancer diagnosis, imaging, and targeting are improved by using biological and non-biological nanomaterials. In tissue engineering, regeneration, and implant engineering nanomaterials provides vast opportunities in curing challenging diseases. Nowadays artificial intelligence is implicated in regenerative medicines and cancer treatments.

\section{AI in Regenerative Medicines and Cancer}

Conventional therapies have limited results in cancer cell treatments. Tumor microenvironment resides a huge population of cancer stem cells that's are self-renewable and regenerate into a tumor. The revolution in $3 \mathrm{D}$ cell culturing, tissue engineering, and cell reprogramming can lead to developing cancer on chips which provide a platform for modeling and analysis of tumor microenvironment. Artificial intelligence improves drug designing, 3D modeling, imaging, and diagnosis of tumors [87]. Artificial intelligence has important applications in remote patient monitoring, medical diagnosis, medical imaging, virtual assistance, hospital management, and risk assessments. Artificial intelligence has a considerable role in surgery, drug delivery, and diagnosis of intestinal and lung cancer by taking advantage of X-rays and mini-robots [88]. Artificial intelligence leaves a major influence on the analysis of genomic information, drug discovery, DNA, or protein sequencing. Artificial neural networks provide help in the development of new hypotheses and treatment strategies. ANN models help in the prediction of the effects of drugs on patients and performing tissue engineering and analysis of their outcomes. AI reduces the cost of tissue engineering and increases therapeutics efficiency. Moreover, it has applications in peptide synthesis, identification of novel antimicrobial drugs and bioactive agents, and monitoring of drug release [89]. According to published literature, AI in conjunction with bioinformatical tools has a synergistic role in diagnostic, prognostic, and predictive accuracies. In recent past years, deep convolutional neural networks are designed for the diagnosis of solid tumors such as thyroid cancer. Certain nanorobots are invented for anatomical uses as drug delivery and imaging of the abdominal cavity. Machine learning exclusively increases the efficiency of cancer treatment and synthesis of artificial tissues and organs [90]. Machine learning, deep learning, advanced computer software, high dimensional databases, and innovative deep learning architecture have a revolutionary impact on cancer stem cells targeting, tissue engineering, regenerative medicines, cell reprogramming, 3D bioprinting, drug discovery, and 3D modeling.

\section{Potential Pitfalls and RiskFactors Associated with Regenerative Medicines}

Regenerative medicines play a critical role in restoring damaged cells or tissues. Stem cells are efficiently used in regenerative medicines but the molecular and cellular mechanisms pathways are hardly described and sometimes efficiency of stem cells to regenerate is limited. The stem cell-based therapies are difficult to administer inpatient body and have limited clinical applications as compare to existing chemical and physical therapies. Moreover, regenerative medicines are not easily handled at the production, development, delivery, and distribution stages [91]. The risk factors of stem cells are including stem cell kinds, differentiation, and proliferation potential of stem cells. These risks are result in the formation of tumors, uncontrolled immune response, and transformation of unpremeditated agents. The mesenchymal stem cell technology is prohibited to regularly use in the clinical laboratory field because of critical potential problems such as the difference between phenotypic character and biological functions of MSCs as well as the shortage of clinical data to support the long-term efficient safety of MSCs. It has been studied that ex vivo technologies hurt mesenchymal stem cells 
including their regenerative properties [92]. Some important features of stem cells are the same as cancer cells such as high replication rate, apoptosis resistance, longer lifetime, similar growth regulators, and control mechanisms. These characters are responsible for malignant tumor formation. The tumor formation of stem cells-based medicines also depends on extrinsic and intrinsic factors such as administration site, manipulation of cells ad in vitro culturing need [93]. Stem cells such as iPSCs are required some genetic modification before clinical applications. Most importantly used viruses to generate iPSCs are retroviruses and lentiviruses, these viruses are genetically modified to encode the genomic DNA that is required for transformation into induced pluripotent stem cells. As the cells have viral integration sites in the genome, these viruses are integrated into the host cells' genome. The use of these viruses is also enhancing the chances of transgene reactivation. For example, reactivation of reprogramming factor as cMyc lead to tumor formation. Hence the use of retroviruses and lentiviruses increases the safety issues [94]. The important risk factors are associated with the administration of stem cell-based clinical products. Mesenchymal stem cells are tissues specific stem cells. It has been reported that in the treatment of myocardial infarction only a few cells are reached to the site of injury when administering intravenously and the engraftment rate is very low when directly apply on the injured part [95].

\section{Future Perspectives and Concerns Challenges}

The stem cells can regenerate damaged tissues and organs. In the current age, as compared to other therapies stem cell therapy has a limited biological substrate for patients. In some chronic diseases the organs become depleted in the stem cells population and difficulties come in contact to meet therapies. In the future by the combination of stem cells with adjuvant therapy, the depleted SCs population can increase for therapeutics [96]. There are several future challenges are related to stem cells one is a hurdle in understanding the complete mechanism of stem cell proliferation. It is suggested that the efficiency of stem cell differentiation makes stem cells more reliable for patients. Moreover, future stem cell therapies also have hurdles. The transplantation of newly generated organs requires the production of millions of cooperative cells [97]. It is previously denoted that cancer therapies should target only cancer stem cells but the normal cancer stem cells are also targeted by chemo or radiotherapies. In addition, cancer stem cells are also attaining drug resistance characters, which it makes more difficult to completely target cancer stem cells. These hurdles force researchers and scientists to design strategies that target cancer stem cells at a molecular level. Notch signaling pathway targeting therapies are used to suppress tumors [98]. The currently organized cancer therapies are insufficient to target cancer cells and elimination of metastatic tumors. For example, breast cancer gene expression is correlated with patient prognosis, and brain cancer stem cells are resistant to radiation through DNA repair mechanisms. Nowadays novel approaches are applicable for targeting cancer cells at the molecular level. In the future, these approaches are further improved by the combination of AI, machine learning, deep learning, nanomaterials, and biomaterials [99]. Cancer stem cells are also targeted at the transcriptional level. RNA interference and miRNAs have a broad-spectrum future for cancer targeting. In the future, at these levels, oncogene pathways are inhibited and increase more chances of cancer treatment.

\section{Conclusion}

Stem cells have huge potential to use in regenerative medicines and tissue engineering. Embryonic stem cells, somatic stem cells, pluripotent stem cells, induced pluripotent stem cells, and mesenchymal stem cells play a decisive role in the regeneration of degrading tissues and organs. Human-induced pluripotent stem cells open a new era of stem cells in the field of regenerative medicines. Because embryonic stem cells are banned, so pluripotency is induced in stem cells by inserting reprogramming genes; cells become induced pluripotent, and in presence of specific and suitable culture these cells can regenerate into any kind of functional and mature cells. Mostly regenerative medicines have revolutionary applications in cancer treatment. Cancer stem cells have similar characters as normal stem cells. Their self-renewable capability made the restriction in targeting. Conventional therapies have limited success in cancer stem cells targeting because the tumor microenvironment has a population of cancer stem cells that's are self-replicated and again tumor formed. Unfortunately, it was not possible to completely target cancer stem cells so new targeting strategies are developed for treatment. In these strategies, cancer stem cells are a target by inhibiting the pathways of oncogenes at the transcriptional level. Smart biomaterials, nanomaterial, miRNAs-based regenerative medicines, AI, and deep learning have an exclusive impact on cancer stem cells targeting. The miRNAs act as biomarkers on cancer cells which provide a new way to design targeting strategies. The most simple, acceptable, and efficient strategy is the use of induced pluripotent stem cells for generating new normal stem cells which replace cancer stem cells. In the future artificial intelligence and artificial neural networks conjunction with advanced bioinformatical tools becomes to improve the targeting, diagnosis, and imaging of tumors.

\section{References}

1. Ilic D, Polak JM. Stem cells in regenerative medicine: introduction. British medical bulletin. 2011; 98: 117-126

2. Griñán-Lisón C, Olivares-Urbano MA, Jiménez G, López-Ruiz E, Del Val C, Morata-Tarifa C, et al. miRNAs as radio-response biomarkers for breast cancer stem cells. Molecular oncology. 2020; 14: 556-570.

3. Ringe J, Kaps C, Burmester GR, Sittinger M. Stem cells for regenerative medicine: advances in the engineering of tissues and organs. Die Naturwissenschaften. 2002; 89: 338-351.

4. Frese L, Dijkman PE, Hoerstrup SP. Adipose Tissue-Derived Stem Cells in Regenerative Medicine. Transfusion medicine and hemotherapy: offizielles Organ der Deutschen Gesellschaft fur Transfusionsmedizin und Immunhamatologie. 2016; 43: 268-274.

5. Di Baldassarre A, Cimetta E, Bollini S, Gaggi G, Ghinassi B. Human-Induced Pluripotent Stem Cell Technology and Cardiomyocyte Generation: Progress and Clinical Applications. Cells. 2018; 7.

6. Hale JS, Otvos B, Sinyuk M, Alvarado AG, Hitomi M, Stoltz K, et al. Cancer stem cell-specific scavenger receptor CD36 drives glioblastoma progression. Stem cells (Dayton, Ohio). 2014; 32: 1746-1758.

7. Siddique HR, Saleem M. Role of BMI1, a stem cell factor, in cancer recurrence and chemoresistance: preclinical and clinical evidences. Stem cells (Dayton, Ohio). 2012; 30: 372-378

8. Yang L, Shi P, Zhao G, Xu J, Peng W, Zhang J, et al. Targeting cancer stem cell pathways for cancer therapy. Signal transduction and targeted therapy. 2020; 5: 8.

9. Arjmand B, Goodarzi P, Mohamadi-Jahani F, Falahzadeh K, Larijani B. 
Personalized Regenerative Medicine. Acta medica Iranica. 2017; 55: 144149.

10. Lin L, Bolund L, Luo Y. Towards Personalized Regenerative Cell Therapy: Mesenchymal Stem Cells Derived from Human Induced Pluripotent Stem Cells. Current stem cell research \& therapy. 2016; 11: 122-130.

11. Larijani B, Aghayan H, Goodarzi P, Mohamadi-Jahani F, Norouzi-Javidan A, Dehpour AR, et al. Clinical Grade Human Adipose Tissue-Derived Mesenchymal Stem Cell Banking. Acta medica Iranica. 2015; 53: 540-546.

12. Pushp P, Nogueira DES, Rodrigues CAV, Ferreira FC, Cabral JMS Gupta MK. A Concise Review on Induced Pluripotent Stem Cell-Derived Cardiomyocytes for Personalized Regenerative Medicine. Stem cell reviews and reports. 2021; $17:$ 748-776.

13. Dzobo K, Thomford NE, Senthebane DA, Shipanga H, Rowe A, Dandara $\mathrm{C}$, et al. Advances in Regenerative Medicine and Tissue Engineering Innovation and Transformation of Medicine. Stem cells international. 2018 2018: 2495848.

14. Bento G, Shafigullina AK, Rizvanov AA, Sardão VA, Macedo MP, Oliveira PJ. Urine-Derived Stem Cells: Applications in Regenerative and Predictive Medicine. Cells. 2020; 9: 573 .

15. Hsu MN, Chang YH, Truong VA, Lai PL, Nguyen TKN, Hu YC. CRISPR technologies for stem cell engineering and regenerative medicine. Biotechnology advances. 2019; 37: 107447.

16. Ban J, Pejatović MM. Nanotechnology approaches for autologous stem cell manipulation in personalized regenerative medicine. Personalized Medicine in Healthcare Systems: Springer. 2019: 45-54.

17. Grath A, Dai G. Direct cell reprogramming for tissue engineering and regenerative medicine. Journal of biological engineering. 2019; 13: 14

18. Desai A, Yan Y, Gerson SL. Concise Reviews: Cancer Stem Cell Targeted Therapies: Toward Clinical Success. Stem cells translational medicine. 2019; 8: $75-81$

19. Xiao W, Gao Z, Duan Y, Yuan W, Ke Y. Notch signaling plays a crucial role in cancer stem-like cells maintaining stemness and mediating chemotaxis in renal cell carcinoma. Journal of experimental \& clinical cancer research: CR 2017; 36: 41.

20. Huang R, Rofstad EK. Cancer stem cells (CSCs), cervical CSCs and targeted therapies. Oncotarget. 2017; 8: 35351-35367.

21. Kolev VN, Wright QG, Vidal CM, Ring JE, Shapiro IM, Ricono J, et al. PI3K/ mTOR dual inhibitor VS-5584 preferentially targets cancer stem cells. Cancer research. 2015; 75: 446-455.

22. Najafi M, Mortezaee K, Majidpoor J. Cancer stem cell (CSC) resistance drivers. Life sciences. 2019; 234: 116781.

23. Wang H, Cui G, Yu B, Sun M, Yang H. Cancer Stem Cell Niche in Colorectal Cancer and Targeted Therapies. Current pharmaceutical design. 2020; 26 1979-1993.

24. Garner KEL, Hull NJ, Sims AH, Lamb R, Clarke RB. The Milk Protein Alpha-Casein Suppresses Triple Negative Breast Cancer Stem Cell Activity Via STAT and HIF-1alpha Signaling Pathways in Breast Cancer Cells and Fibroblasts. Journal of mammary gland biology and neoplasia. 2019; 24: 245256

25. Lou H, Dean M. Targeted therapy for cancer stem cells: the patched pathway and ABC transporters. Oncogene. 2007; 26: 1357-1360.

26. Prabavathy D, Swarnalatha $Y$, Ramadoss N. Lung cancer stem cells-origin, characteristics and therapy. Stem cell investigation. 2018; 5: 6.

27. Vijay GV, Zhao N, Den Hollander P, Toneff MJ, Joseph R, Pietila M, et al. GSK3 $\beta$ regulates epithelial-mesenchymal transition and cancer stem cell properties in triple-negative breast cancer. Breast cancer research: BCR. 2019; $21: 37$

28. Marzagalli M, Fontana F, Raimondi M, Limonta P. Cancer Stem Cells-Key Players in Tumor Relapse. Cancers. 2021; 13: 376.

29. Stem J, Flickinger JC Jr., Merlino D, Caparosa EM, Snook AE, Waldman
SA. Therapeutic targeting of gastrointestinal cancer stem cells. Regenerative medicine. 2019; 14: 331-343.

30. June CH, O'Connor RS, Kawalekar OU, Ghassemi S, Milone MC. CAR T cell immunotherapy for human cancer. Science (New York, NY). 2018; 359: 1361-1365

31. Pietra G, Manzini C, Vitale M, Balsamo M, Ognio E, Boitano M, et al. Natural killer cells kill human melanoma cells with characteristics of cancer stem cells. International immunology. 2009; 21: 793-801.

32. Hao J, Fan W, Li Y, Tang R, Tian C, Yang Q, et al. Melatonin synergizes BRAF-targeting agent vemurafenib in melanoma treatment by inhibiting iNOS/hTERT signaling and cancer-stem cell traits. Journal of experimental \& clinical cancer research: CR. 2019; 38: 48.

33. Ghasemi S, Xu S, Nabavi SM, Amirkhani MA, Sureda A, Tejada S, et al Epigenetic targeting of cancer stem cells by polyphenols (cancer stem cells targeting). Phytotherapy research: PTR. 2021.

34. Schram AM, O'Reilly EM, Somwar R, Benayed R, Shameem S, Chauhan T, et al. Abstract PR02: Clinical proof of concept for MCLA-128, a bispecific HER2/3 antibody therapy, in NRG1 fusion-positive cancers. AACR. 2019.

35. Casaos J, Gorelick NL, Huq S, Choi J, Xia Y, Serra R, et al. The Use of Ribavirin as an Anticancer Therapeutic: Will It Go Viral? Molecular cancer therapeutics. 2019; 18: 1185-1194.

36. Udhane V, Maranto C, Hoang DT, Gu L, Erickson A, Devi S, et al Enzalutamide-Induced Feed-Forward Signaling Loop Promotes TherapyResistant Prostate Cancer Growth Providing an Exploitable Molecular Target for Jak2 Inhibitors. Molecular cancer therapeutics. 2020; 19: 231-246.

37. Ho AL, Brana I, Haddad R, Bauman J, Bible K, Oosting S, et al. Tipifarnib in Head and Neck Squamous Cell Carcinoma With HRAS Mutations. Journal of clinical oncology: official journal of the American Society of Clinical Oncology. 2021: 39: 1856-1864.

38. Krop I, Demuth T, Guthrie T, Wen PY, Mason WP, Chinnaiyan P, et al. Phase pharmacologic and pharmacodynamic study of the gamma secretase (Notch) inhibitor MK-0752 in adult patients with advanced solid tumors. Journal of clinical oncology: official journal of the American Society of Clinical Oncology. 2012; 30: 2307-2313

39. Chiorean EG, LoRusso P, Strother RM, Diamond JR, Younger A, Messersmith WA, et al. A Phase I First-in-Human Study of Enoticumab (REGN421), a Fully Human Delta-like Ligand 4 (D\|l4) Monoclonal Antibody in Patients with Advanced Solid Tumors. Clinical cancer research: an official journal of the American Association for Cancer Research. 2015; 21: 2695-2703.

40. López-Guerra M, Xargay-Torrent S, Fuentes P, Roldán J, GonzálezFarré B, Rosich L, et al. Specific NOTCH1 antibody targets DLL4-induced proliferation, migration, and angiogenesis in NOTCH1-mutated CLL cells. Oncogene. 2020; 39: 1185-1197.

41. Okazaki H, Sato S, Koyama K, Morizumi S, Abe S, Azuma M, et al. The novel inhibitor PRI-724 for Wnt/ß-catenin/CBP signaling ameliorates bleomycininduced pulmonary fibrosis in mice. Experimental lung research. 2019; 45: 188-199.

42. Minami H, Fujiwara Y, Muro K, Sato M, Moriya A. Phase I study of BGT226 a pan-PI3K and mTOR inhibitor, in Japanese patients with advanced solid cancers. Cancer chemotherapy and pharmacology. 2019; 84: 337-343.

43. Takei J, Kaneko MK, Ohishi T, Hosono H, Nakamura T, Yanaka M, et al A defucosylated anti-CD44 monoclonal antibody 5-mG2a-f exerts antitumor effects in mouse xenograft models of oral squamous cell carcinoma. Oncology reports. 2020; 44: 1949-1960.

44. He SF, Wang W, Ren $\mathrm{H}$, Zhao LJ, Qi ZT. Interferon alpha and ribavirin collaboratively regulate p38 mitogen-activated protein kinase signaling in hepatoma cells. Cytokine. 2013; 61: 801-807.

45. Fang-Fang Z, You Y, Wen-Jun L. Progress in research on childhood T-cell acute lymphocytic leukemia, Notch1 signaling pathway, and its inhibitors: A review. Bosnian journal of basic medical sciences. 2021; 21: 136-144.

46. McCaw TR, Inga $\mathrm{E}$, Chen $\mathrm{H}$, Jaskula-Sztul $\mathrm{R}$, Dudeja $\mathrm{V}$, Bibb JA, et al. Gamma secretase inhibitors in cancer: a current perspective on clinical 
performance. 2021; 26: e608-e621.

47. Watanabe M, Otake R, Kozuki R, Toihata T, Takahashi K, Okamura A, et al Recent progress in multidisciplinary treatment for patients with esophageal cancer. Surgery today. 2020; 50: 12-20.

48. Davis SL, Cardin DB, Shahda S, Lenz H-J, Dotan E, O'Neil BH, et al. A phase 1 b dose escalation study of Wnt pathway inhibitor vantictumab in combination with nab-paclitaxel and gemcitabine in patients with previously untreated metastatic pancreatic cancer. 2020; 38: 821-830

49. Lee HJ, Choi MY, Siddiqi T, Barrientos JC, Wierda WG, Isufi I, et al. Phase $1 / 2$ study of cirmtuzumab and ibrutinib in mantle cell lymphoma (MCL) or chronic lymphocytic leukemia (CLL). Wolters Kluwer Health. 2021.

50. Niemann CU, Levin MD, Dubois J, Kersting S, Enggaard L, Veldhuis GJ et al. Venetoclax and ibrutinib for patients with relapsed/refractory chronic lymphocytic leukemia. Blood. 2021; 137: 1117-1120.

51. Lee SWL, Paoletti C, Campisi M, Osaki T, Adriani G, Kamm RD, et al. MicroRNA delivery through nanoparticles. Journal of controlled release: official journal of the Controlled Release Society. 2019; 313: 80-95.

52. Khan AQ, Ahmed El, Elareer NR, Junejo K, Steinhoff M, Uddin S. Role of miRNA-Regulated Cancer Stem Cells in the Pathogenesis of Human Malignancies. Cells. 2019; 8.

53. Kuang Y, Cao J, Xu F, Chen Y. Duplex-Specific Nuclease-Mediated Amplification Strategy for Mass Spectrometry Quantification of MiRNA-200c in Breast Cancer Stem Cells. Analytical chemistry. 2019; 91: 8820-8826.

54. Ruggieri V, Russi S, Zoppoli P, La Rocca F, Angrisano T, Falco G, et al. The Role of MicroRNAs in the Regulation of Gastric Cancer Stem Cells: A MetaAnalysis of the Current Status. Journal of clinical medicine. 2019; 8.

55. Xiao WS, Li DF, Tang YP, Chen YZ, Deng WB, Chen J, et al. Inhibition of epithelial-mesenchymal transition in gastric cancer cells by miR-711-mediated downregulation of CD44 expression. Oncology reports. 2018; 40: 2844-2853.

56. Shirmohamadi M, Eghbali E, Najjary S, Mokhtarzadeh A, Kojabad AB Hajiasgharzadeh K, et al. Regulatory mechanisms of microRNAs in colorectal cancer and colorectal cancer stem cells. Journal of cellular physiology. 2020; 235: 776-789.

57. Yang Z, Zhao N, Cui J, Wu H, Xiong J, Peng T. Exosomes derived from cancer stem cells of gemcitabine-resistant pancreatic cancer cells enhance drug resistance by delivering miR-210. Cellular oncology (Dordrecht). 2020 43: 123-136.

58. Aghajani M, Mansoori B, Mohammadi A, Asadzadeh Z, Baradaran B. New emerging roles of CD133 in cancer stem cell: Signaling pathway and miRNA regulation. Journal of cellular physiology. 2019; 234: 21642-21661.

59. Tutar L, Tutar E, Özgür A, Tutar Y. Therapeutic Targeting of microRNAs in Cancer: Future Perspectives. Drug development research. 2015; 76: 382 388.

60. Laneve P, Di Marcotullio L, Gioia U, Fiori ME, Ferretti E, Gulino A, et al. The interplay between microRNAs and the neurotrophin receptor tropomyosinrelated kinase $\mathrm{C}$ controls proliferation of human neuroblastoma cells. Proceedings of the National Academy of Sciences of the United States of America. 2007; 104: 7957-7962.

61. Ma L, Teruya-Feldstein J, Weinberg RA. Tumour invasion and metastasis initiated by microRNA-10b in breast cancer. Nature. 2007; 449: 682-688.

62. Hossain A, Kuo MT, Saunders GF. Mir-17-5p regulates breast cancer cell proliferation by inhibiting translation of AIB1 mRNA. Molecular and cellular biology. 2006; 26: 8191-8201.

63. Cimmino A, Calin GA, Fabbri M, Iorio MV, Ferracin M, Shimizu M, et al. miR-15 and miR-16 induce apoptosis by targeting BCL2. Proceedings of the National Academy of Sciences of the United States of America. 2005; 102: 13944-13949.

64. Dews M, Homayouni A, Yu D, Murphy D, Sevignani C, Wentzel E, et al. Augmentation of tumor angiogenesis by a Myc-activated microRNA cluster. Nature genetics. 2006; 38: 1060-1065

65. Meng F, Henson R, Wehbe-Janek H, Ghoshal K, Jacob ST, Patel T.
MicroRNA-21 regulates expression of the PTEN tumor suppressor gene in human hepatocellular cancer. Gastroenterology. 2007; 133: 647-658.

66. Welch C, Chen Y, Stallings RL. MicroRNA-34a functions as a potential tumor suppressor by inducing apoptosis in neuroblastoma cells. Oncogene. 2007; 26: 5017-5022.

67. Saito Y, Liang G, Egger G, Friedman JM, Chuang JC, Coetzee GA, et al. Specific activation of microRNA-127 with downregulation of the protooncogene BCL 6 by chromatin-modifying drugs in human cancer cells. Cancer cell. 2006; 9: 435-443.

68. Adams BD, Furneaux $\mathrm{H}$, White BA. The micro-ribonucleic acid (miRNA) miR206 targets the human estrogen receptor-alpha (ERalpha) and represses ERalpha messenger RNA and protein expression in breast cancer cell lines. Molecular endocrinology (Baltimore, Md). 2007; 21: 1132-1147.

69. Galardi S, Mercatelli N, Giorda E, Massalini S, Frajese GV, Ciafrè SA, et al miR-221 and miR-222 expression affects the proliferation potential of human prostate carcinoma cell lines by targeting p27Kip1. The Journal of biological chemistry. 2007; 282: 23716-23724.

70. Brokesh AM, Gaharwar AK. Inorganic Biomaterials for Regenerative Medicine. ACS applied materials \& interfaces. 2020; 12: 5319-5344.

71. Su Y, Cockerill I, Wang Y, Qin YX, Chang L, Zheng Y, et al. Zinc-Based Biomaterials for Regeneration and Therapy. Trends in biotechnology. 2019; 37: 428-441.

72. Sultankulov B, Berillo D, Sultankulova K, Tokay T, Saparov A. Progress in the Development of Chitosan-Based Biomaterials for Tissue Engineering and Regenerative Medicine. Biomolecules. 2019; 9: 470.

73. Moztarzadeh S, Mottaghy K, Sefat F, Samadikuchaksaraei A, Mozafar M. Nanoengineered biomaterials for lung regeneration. Nanoengineered Biomaterials for Regenerative Medicine: Elsevier. 2019: 305-323.

74. Ogueri KS, Ogueri KS, Allcock HR, Laurencin CT. Polyphosphazene polymers: The next generation of biomaterials for regenerative engineering and therapeutic drug delivery. Journal of vacuum science and technology B, Nanotechnology \& microelectronics: materials, processing, measurement, \& phenomena: JVST B. 2020; 38: 030801.

75. Urbanska AM, Sefat F, Yousaf S, Kargozar S, Milan PB, Mozafari M. Nanoengineered biomaterials for intestine regeneration. Nanoengineered biomaterials for regenerative medicine: Elsevier. 2019: 363-378.

76. Facklam AL, Volpatti LR, Anderson DG. Biomaterials for Personalized Cell Therapy. Advanced materials (Deerfield Beach, Fla). 2020; 32: e1902005.

77. Midgley AC, Wei Y, Li Z, Kong D, Zhao Q. Nitric-Oxide-Releasing Biomateria Regulation of the Stem Cell Microenvironment in Regenerative Medicine. Advanced materials (Deerfield Beach, Fla). 2020; 32: e1805818.

78. Chen Q, Chen M, Liu Z. Local biomaterials-assisted cancer immunotherapy to trigger systemic antitumor responses. Chemical Society reviews. 2019; 48: 5506-5526.

79. dos Santos J, de Oliveira RS, de Oliveira TV, Velho MC, Konrad MV, da Silva GS, et al. 3D Printing and Nanotechnology: A Multiscale Alliance in Personalized Medicine. 2021; 31: 2009691.

80. Khan AU, Khan M, Cho MH, Khan MM. Selected nanotechnologies and nanostructures for drug delivery, nanomedicine and cure. Bioprocess and biosystems engineering. 2020; 43: 1339-1357.

81. Purushothaman AE, Thakur K, Kandasubramanian B. Development of highly porous, Electrostatic force assisted nanofiber fabrication for biological applications. 2020; 69: 477-504.

82. Mariappan NJB, Journal P. Recent trends in Nanotechnology applications in surgical specialties and orthopedic surgery. 2019; 12: 1095-1127.

83. Deng C, Xu C, Zhou Q, Cheng Y. Advances of nanotechnology in osteochondral regeneration. Wiley interdisciplinary reviews Nanomedicine and nanobiotechnology. 2019; 11: e1576.

84. Fang J, Hsueh YY, Soto J, Sun W, Wang J, Gu Z, et al. Engineering Biomaterials with Micro/Nanotechnologies for Cell Reprogramming. ACS nano. 2020; 14: 1296-1318 
85. Kumar S, Nehra M, Kedia D, Dilbaghi N, Tankeshwar K, Kim KH Nanotechnology-based biomaterials for orthopaedic applications: Recent advances and future prospects. Materials science \& engineering C, Materials for biological applications. 2020; 106: 110154.

86. Kargozar S, Baino F, Hamzehlou S, Hamblin MR, Mozafari M. Nanotechnology for angiogenesis: opportunities and challenges. Chemical Society reviews. 2020; 49: 5008-5057.

87. Fetah KL, DiPardo BJ, Kongadzem EM, Tomlinson JS, Elzagheid A, Elmusrat $\mathrm{M}$, et al. Cancer Modeling-on-a-Chip with Future Artificial Intelligence Integration. Small (Weinheim an der Bergstrasse, Germany). 2019; 15: e1901985

88. Dwivedi A, Dwivedi SS, Tariq MR, Qiu X, Hong S, Xin Y, et al. Scope of Artificial Intelligence in Medicine. 2020; 8: 137-140.

89. Hassanzadeh P, Atyabi F, Dinarvand R. The significance of artificial intelligence in drug delivery system design. Advanced drug delivery reviews. 2019; 151-152: 169-190.

90. Huang S, Yang J, Fong S, Zhao Q. Artificial intelligence in cancer diagnosis and prognosis: Opportunities and challenges. Cancer letters. 2020; 471: 61 71.

91. Umemura MJBH. Challenging the problem of 'fit': Advancing the regenerative medicine industries in the United States, Britain and Japan. 2019; 61: 456-

92. Herberts CA, Kwa MS, Hermsen HP. Risk factors in the development of stem cell therapy. Journal of translational medicine. 2011; 9: 29.
93. Li HC, Stoicov C, Rogers AB, Houghton J. Stem cells and cancer: evidence for bone marrow stem cells in epithelial cancers. World journal of gastroenterology. 2006; 12: 363-371.

94. Kaji K, Norrby K, Paca A, Mileikovsky M, Mohseni P, Woltjen K. Virus-free induction of pluripotency and subsequent excision of reprogramming factors. Nature. 2009; 458: 771-775.

95. Menasché P. Stem cell therapy for heart failure: are arrhythmias a real safety concern? Circulation. 2009; 119: 2735-2740

96. Brunt KR, Weisel RD, Li RK. Stem cells and regenerative medicine - future perspectives. Canadian journal of physiology and pharmacology. 2012; 90 327-335.

97. Zakrzewski W, Dobrzyński M, Szymonowicz M, Rybak Z. Stem cells: past present, and future. Stem cell research \& therapy. 2019; 10: 68.

98. Kaur S, Singh G, Kaur K. Cancer stem cells: an insight and future perspective. Journal of cancer research and therapeutics. 2014; 10: 846-852.

99. Shukla G, Khera HK, Srivastava AK, Khare P, Patidar R, Saxena R. Therapeutic Potential, Challenges and Future Perspective of Cancer Stem Cells in Translational Oncology: A Critical Review. Current stem cell research \& therapy. 2017: 12: 207-224. 